\title{
Description and Simulation of OFDM Reception Process
}

\author{
S. Sadinov ${ }^{1}$, P. Daneva ${ }^{1}$ and P. Kogias ${ }^{2, *}$ \\ ${ }^{I}$ Technical University of Gabrovo, Bulgaria \\ ${ }^{2}$ Department of Electrical Engineering, Eastern Macedonia and Thrace Institute of Technology, Greece
}

Received 2 June 2014; Accepted 2 July 2014

\begin{abstract}
Orthogonal Frequency Division Multiplexing (OFDM) is becoming the chosen modulation technique for modern communications because of its resistance to inter-symbol interference (ISI) and its low-complexity implementation for high-speed system compared to traditional single technique. The purpose of this paper is to describe and simulate the basic processing involved in the receiver of the OFDM system. As an example, one of the proposed OFDM signals of the Digital Video Broadcasting - Terrestrial (DVB - T) standard is used. Matlab simulation results for $2 \mathrm{k}$ mode of the standard are presented.
\end{abstract}

Keywords: DVB-T, FFT, Matlab, OFDM

\section{Introduction}

Orthogonal Frequency Division Multiplexing (OFDM) is a special form of Multicarrier modulation which is particularly suited for transmission over a dispersive channel. Here the different carriers are orthogonal to each other, that is, they are totally independent of one another. OFDM is a wideband modulation scheme that is designed to cope with the problems of the multipath reception. Essentially, the wideband frequency selective fading channel is divided into many narrow-band sub channels. If the number of sub channels is high enough, each sub channel could be considered as flat. This is because many narrowband overlapping digital signals in parallel are transmitted, inside one wide band. Increasing the number of parallel transmission channels reduces the data rate that each individual carrier must convey, and that lengthens the symbol period. Therefore the delay time of reflected waves is suppressed to within 1 symbol time.

The development of OFDM systems can be divided into three parts that are Frequency Division Multiplexing, Multicarrier Communication and Orthogonal Frequency Division Multiplexing [1], [2], [3]. Frequency Division Multiplexing is a form of signal multiplexing which involves assigning non overlapping frequency ranges or channels to different signals or to each user of a medium. A gap or guard band is left between each of these channels to ensure that the signal of one channel does not overlap with the signal from an adjacent one. Multicarrier Communication involves splitting of the signal to give a number of signals over that frequency range. Each of these signals are individually modulated and transmitted over the channel. At the receiver end, these signals are demodulated and recombined to obtain the original signal.
In many books and scientific works for OFDM systems, such as [2], [3], [4], [5], [6], [7], etc., the basic processing involved in the generation and transmission of an OFDM is examined in detail. At the same time, considerably less attention is paid to the examination of the reception signal processing. Thus more work needs to be done on studying OFDM reception. As well known, the design of an OFDM receiver is open, because there are only transmission standards. With an open receiver design, most of the researches and innovations are done in the receiver. The purpose of this paper is to describe and simulate each of the steps involved in the OFDM reception process since the examination of this process is essential for the design of OFDM systems.

\section{Description and Simulation of OFDM Reception Process}

To describe and simulate the OFDM reception process, an appropriate receiver model and simulation resources must be used.

An OFDM receiver model can be shown as Fig. 1.

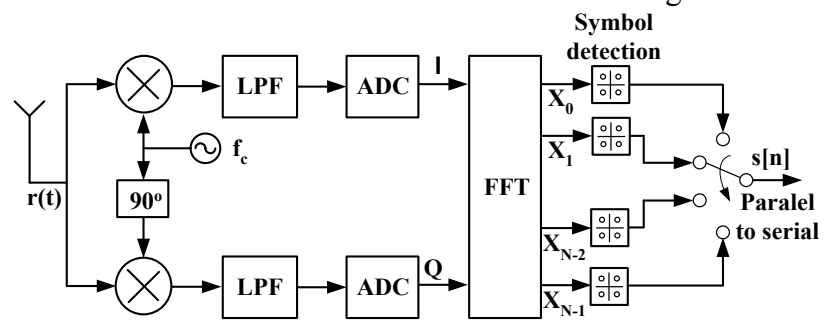

Fig. 1. An idealized OFDM receiver model

In the above model $r(t)$ is the input receiver signal. 
Resources for the simulation of the OFDM reception are included in Matlab in functions of the Communication and Signal Processing Toolboxes. These resources enable a complete analysis of received data. To simulate the basic processing involved in the reception of an OFDM signal, as an example, one of the proposed OFDM signals of the Digital Video Broadcasting - Terrestrial (DVB - T) standard [8] is used. Matlab simulation is implemented for the $2 \mathrm{k}$ mode of the DVB - T standard. The specific numerical values for the OFDM parameters for the $2 \mathrm{k}$ mode are given in Table 1 [8], [9].

Table 1. Numerical values for the OFDM parameters for the $2 \mathrm{k}$ mode

\begin{tabular}{|c|c|c|c|c|}
\hline Parameter & \multicolumn{4}{|c|}{ 2k mode } \\
\hline Elementary period $\mathbf{T}, \boldsymbol{\mu s}$ & \multicolumn{4}{|c|}{$7 / 64$} \\
\hline Number of carriers $\mathbf{K}$ & \multicolumn{4}{|c|}{1705} \\
\hline Value of carrier number $\mathbf{K m i n}$ & \multicolumn{4}{|c|}{$\mathbf{0}$} \\
\hline Value of carrier number $\mathbf{K} \mathbf{m a x}$ & \multicolumn{4}{|c|}{1704} \\
\hline Duration of symbol part $\mathbf{T}_{\mathbf{U}}, \boldsymbol{\mu} \mathbf{s}$ & \multicolumn{4}{|c|}{$\begin{array}{c}2048 \times T \\
224\end{array}$} \\
\hline Carrier spacing $\mathbf{1} / \mathbf{T}_{\mathbf{U}}, \mathrm{Hz}$ & \multicolumn{4}{|c|}{4464} \\
\hline $\begin{array}{l}\text { Spacing between carriers Kmin } \\
\text { and }\end{array}$ & \multicolumn{4}{|c|}{7.612} \\
\hline $\operatorname{Kmax}(\mathbf{K}-1) / \mathbf{T}_{\mathbf{U}}, \mathrm{MHz}$ & & & & \\
\hline Allowed guard interval $\Delta / \mathbf{T}_{\mathbf{U}}$ & $1 / 4$ & $1 / 8$ & $1 / 16$ & $1 / 32$ \\
\hline Duration of guard interval $\boldsymbol{\Delta}, \boldsymbol{\mu} \mathbf{s}$ & 56 & 28 & 14 & 7 \\
\hline $\begin{array}{l}\text { Symbol duration } \\
\mathbf{T s}=\Delta+\mathbf{T} \mathbf{u}, \boldsymbol{\mu s}\end{array}$ & 280 & 252 & 238 & 231 \\
\hline
\end{tabular}

When the receiver senses the signal, the Radio Frequency (RF) signal is first down-converted. Simulation results of this operation are shown in Fig. 2 and Fig. 3.
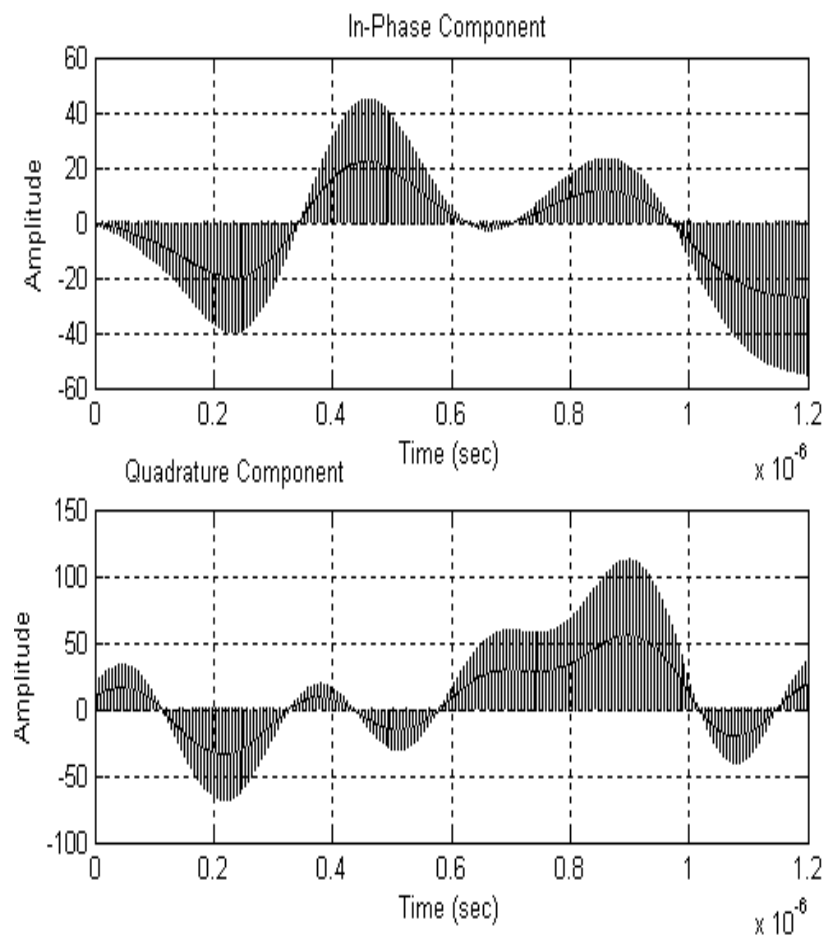

Fig. 2. Time response of down - converted signal

Fig. 2 displays the In-phase (I) and quadrature (Q) components of a down - converted signal as a function of time.

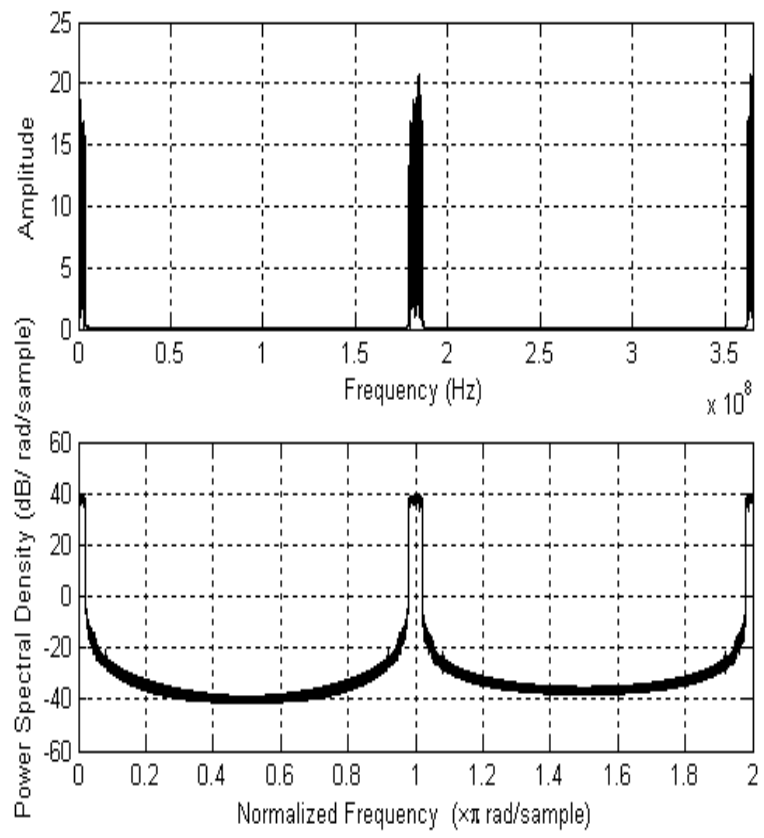

Fig. 3. Frequency response of down - converted signal

It can be seen from Fig.3 that the down - conversion process creates signals centered on $2 f_{c}$, where $f_{c}$ is the central frequency of the RF signal. To reject these signals, i.e., to generate a continuous time baseband signal, Low Pass Filters (LPFs) are used. These filters are Butterworth filters of order 3 and cut-off frequency of $2 \pi f_{c}$.

The in-phase and quadrature components of the baseband signal in the time domain are shown in Fig. 4. It follows from Fig. 4 that the continuous time baseband signal is a copy of the reconstruction filter's output signal at the transmitter [10].
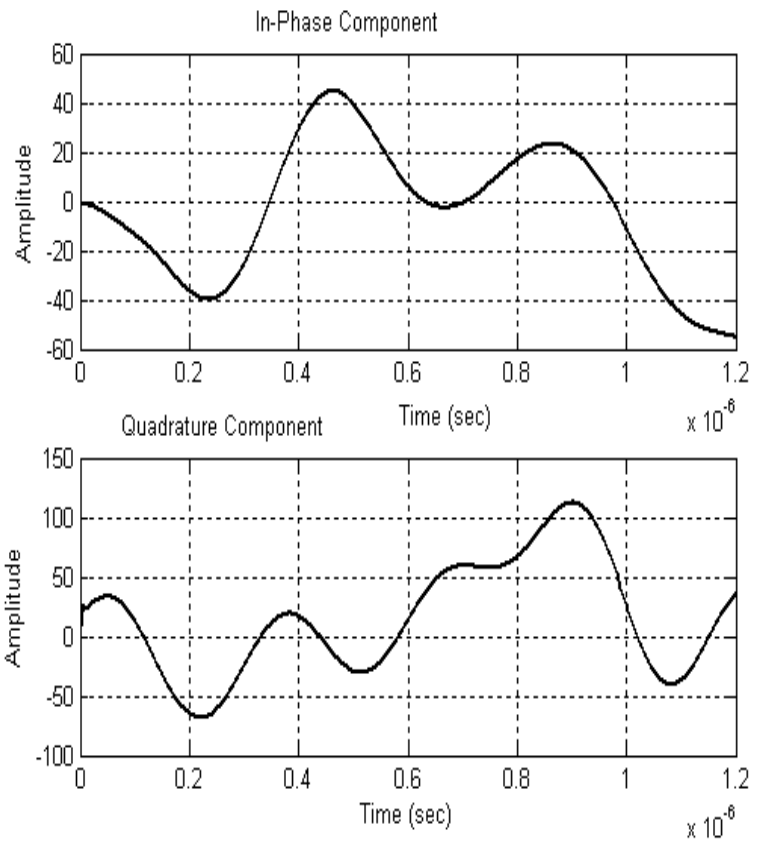

Fig. 4. Filtered signal time response

The filter's output signal versus the frequency is presented in Fig. 5. According to the simulation results it is 
possible to conclude, that the filtering is performed as expected since is left with only the baseband spectrum.
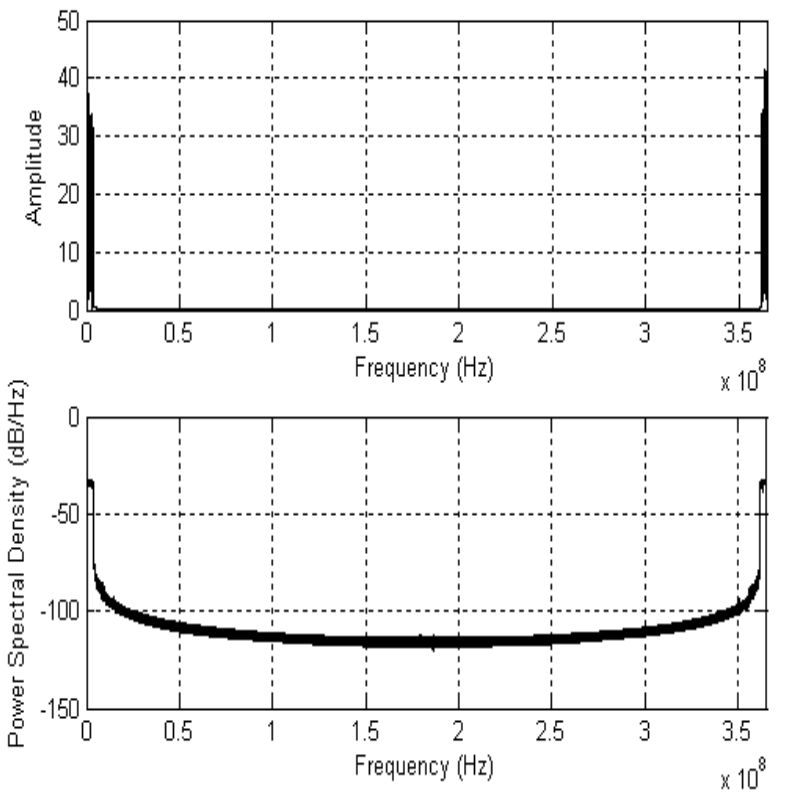

Fig. 5. Filtered signal frequency response

The next step of the reception signal processing is the sampling of the continuous time baseband signal. Each one of the in-phase and quadrature component is sampled using Analog - to - Digital Converter (ADC). The sampling frequency is chosen to be $F_{S}=2 / T$, where $T$ is the elementary period for a baseband signal. In Fig. 6 and Fig. 7 it can be observed the simulation results of this operation and that the discrete time baseband signal uses $T / 2$ as its time period. Moreover, from the comparison of the frequency response shown in Fig.7 with the analogue frequency response of the Inverse Fast Fourier Transform (IFFT) implementation [10] at the transmitter of the OFDM system can be established that there is a slight difference between the transmitted and received OFDM signals. The presence of this difference is due to the delay produced by the filtering operation. The total delay produced by the reconstruction or $\mathrm{D} / \mathrm{A}$, and demodulation filters is about $t_{d}=64 / R_{S}$, where $R_{S}$ is the simulation period.
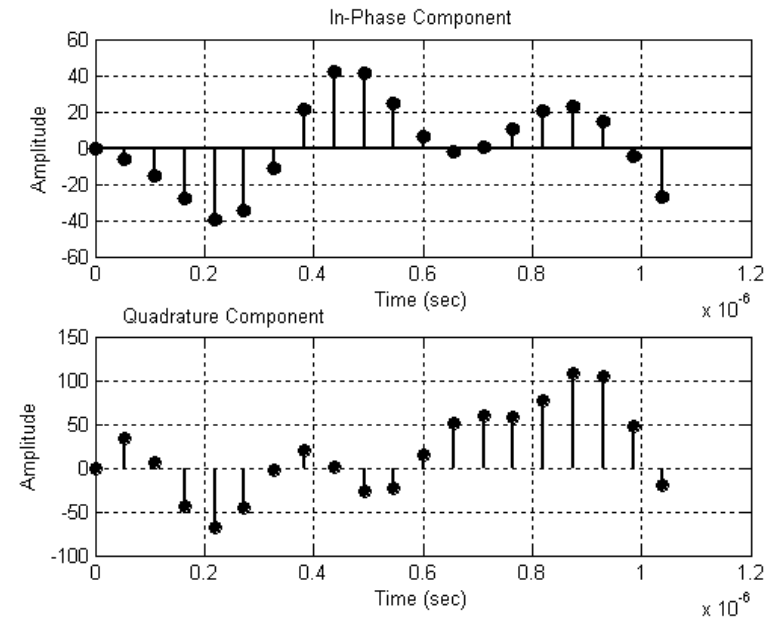

Fig. 6. Time response of sampled signal
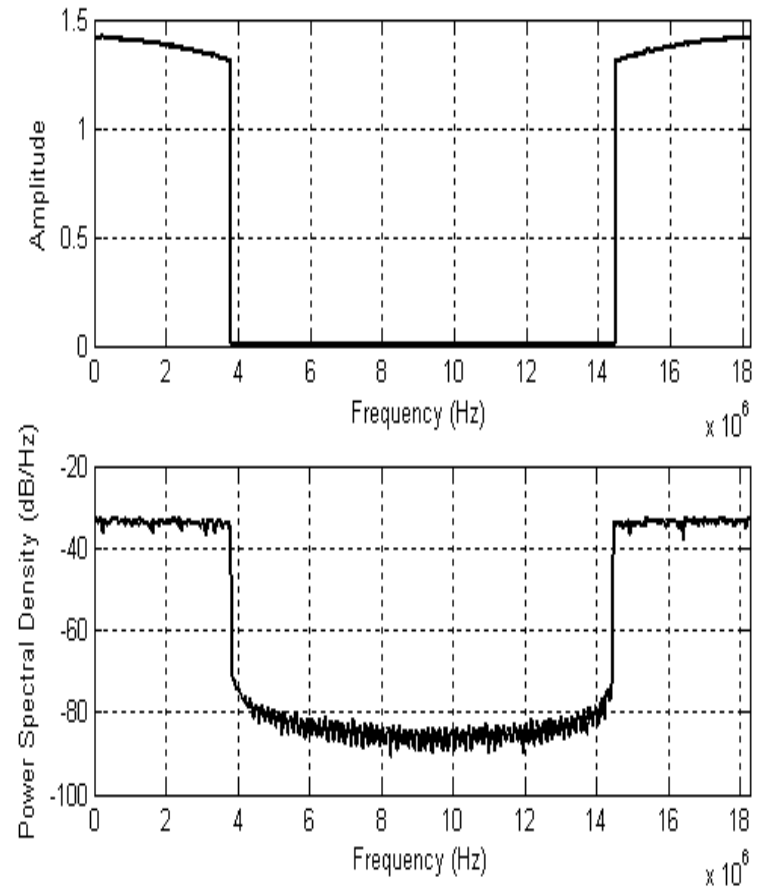

Fig. 7. Frequency response of sampled signal

In the next step of the signal processing, the time domain samples are converted into a frequency domain representation, using a forward Fast Fourier Transform (FFT). The magnitudes of the frequency components correspond to the original data. As well known [3], [11], for an OFDM system that has the same sample rate for both the transmitter and receiver, it must use the same FFT size at both the receiver and transmitted OFDM signal in order to maintain subcarrier orthogonality. Because of the OFDM spectrum centering on $f_{c}$, a $2 \mathrm{~N}$ - point, instead of $\mathrm{N}$-point, FFT processing is recommended [5], [12]. As can be seen in Table 1, the OFDM symbol duration, $\mathrm{T}_{\mathrm{U}}$, is specified for $\mathrm{N}=2048$. Therefore, a 4096 - FFT is used. In this case, the first $2 \mathrm{~N}$ - points of the discrete time OFDM waveform are input into a $2 \mathrm{~N}$ - points FFT, taking the first $\mathrm{N}$ output complex data samples which are equivalent to the output inphase and quadrature matched filters. The output of the FFT in frequency domain is given by

$X_{k}=\sum_{n=0}^{N-1} x_{n} e^{-j 2 \pi k n / N}$

where $\mathrm{X}_{\mathrm{k}}$ is the frequency output of the FFT at $\mathrm{k}^{\text {th }}$ point $(\mathrm{k}=0,1, \ldots, \mathrm{N}-1)$ and $\mathrm{x}_{\mathrm{n}}$ is the time sample at $\mathrm{n}^{\text {th }}$ point with $\mathrm{n}=0,1, \ldots, \mathrm{N}-1$.

The received constellation diagram is illustrated in Fig. 8. Simulation is carried out for quadrature amplitude modulation (QAM) as a modulation technique, which in the particular case is 4-QAM.

After FFT processing, the received signal must be demodulated, i.e., demapped. The function of this process is to convert the complexes valued constellations points to symbols. This is done using 4 - QAM signal demapping. The received constellation in form of 4 - QAM demapped symbols is shown in Fig. 9. 
It is visible in Fig. 8 and Fig. 9 that the demapping process is correctly implemented.

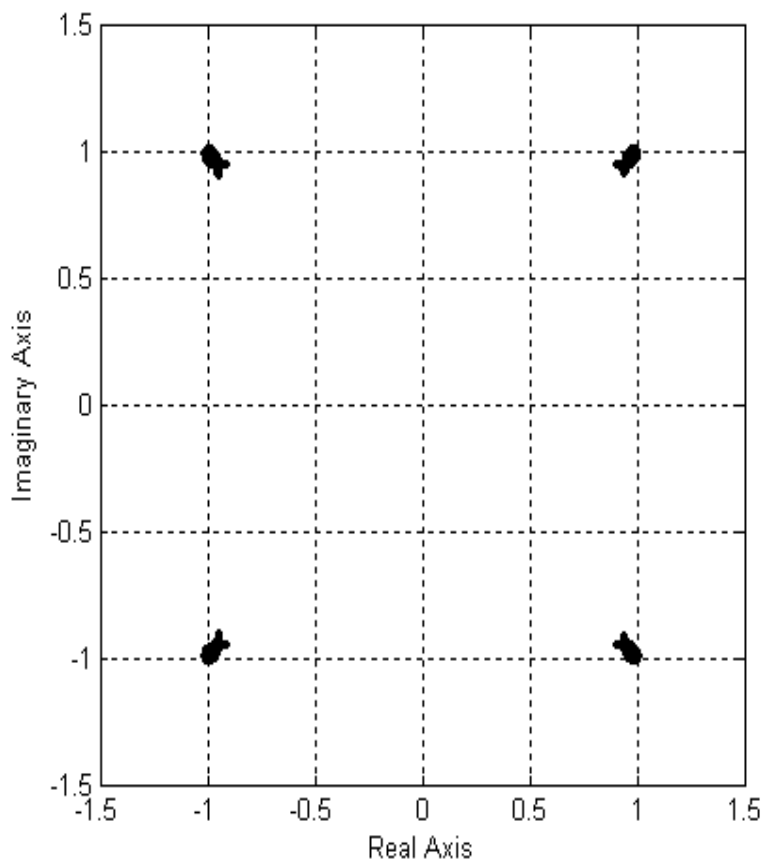

Fig. 8. 4-QAM constellation before 4-QAM demapping

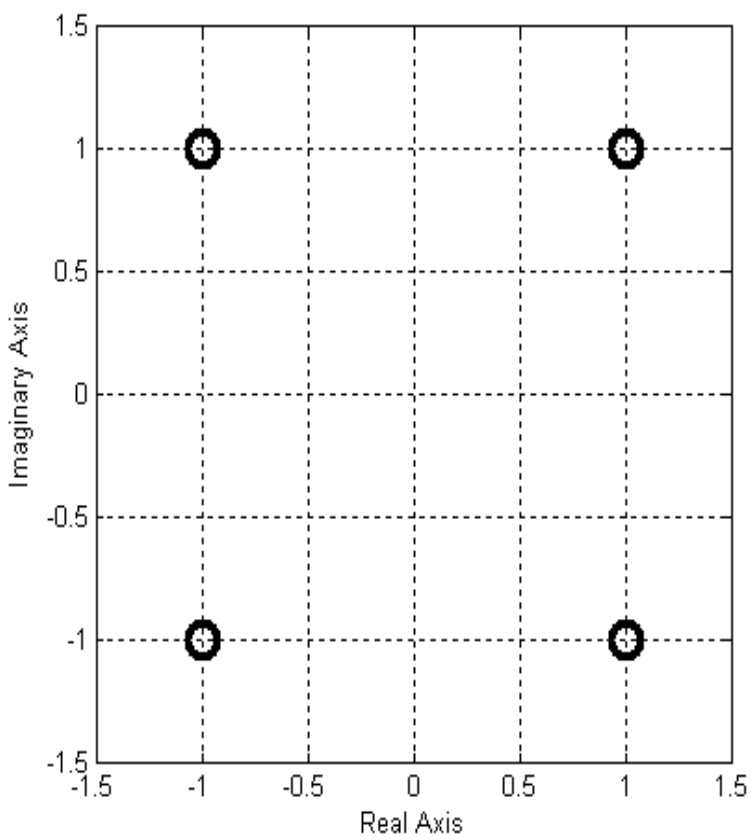

Fig. 9. 4-QAM constellation after 4-QAM demapping

The received constellation from Fig. 9 corresponds to the original one.

In the real case must take into account the influence of the channel noise on the received constellation. If the signal is transmitted over an Additive White Gaussian Noise (AWGN) channel, the type of the constellation depends on the signal - noise - ratio (SNR) of the channel. Simulation results for SNR values of $2 \mathrm{~dB}$ and $12 \mathrm{~dB}$ are shown in Fig. 10 and Fig. 11, respectively.

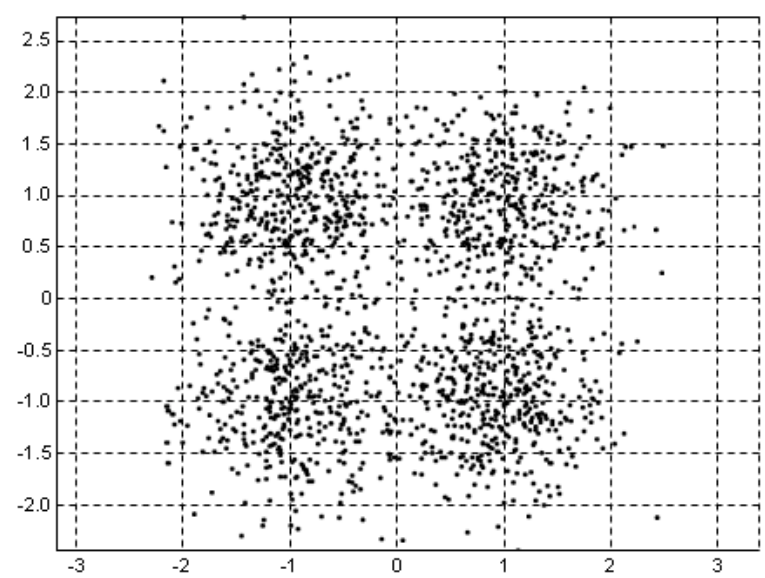

Fig. 10. Received $4-Q A M$ constellation for $S N R=2 d B$

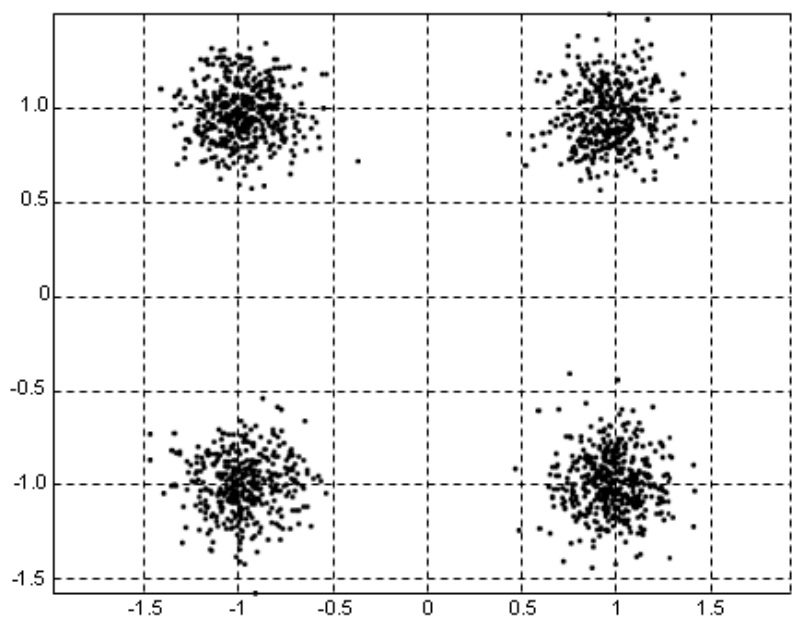

Fig. 11. Received 4-QAM constellation for $S N R=12 d B$

It is clear that as the SNR is increased the received constellation gets less affected by the noise, hence there will be less errors. On the other hand, for low values of SNR there are ISI introduced by the noise at the receiver side. This is presented in Fig. 12, Fig. 13 and Fig. 14.
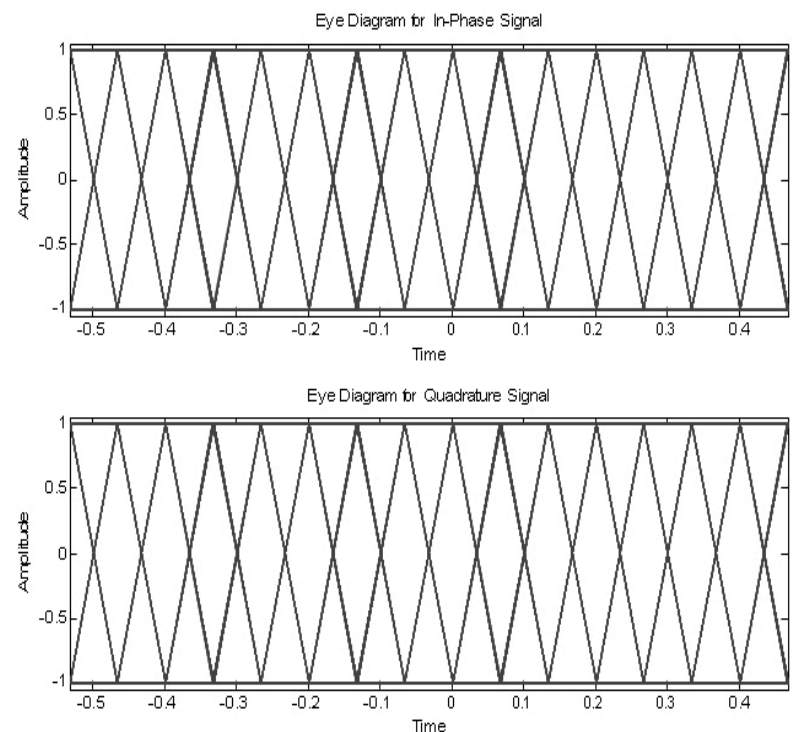

Fig. 12. Eye pattern for the received constellation in an ideal channel 

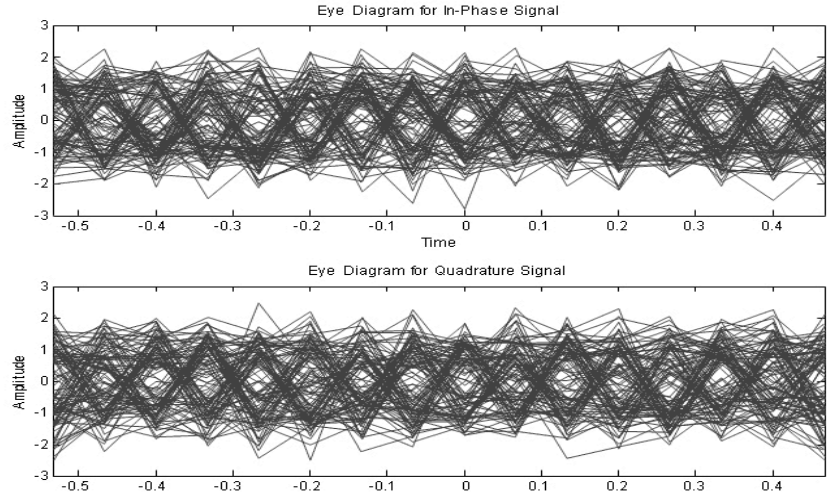

Fig. 13. Eye pattern for the received constellation for $\mathrm{SNR}=2 \mathrm{~dB}$
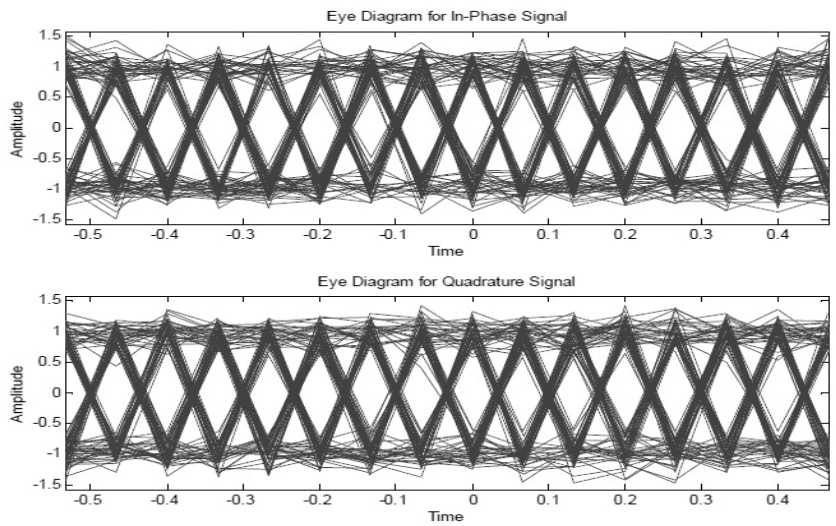

Fig. 14. Eye pattern for the received constellation for $\mathrm{SNR}=12 \mathrm{~dB}$
It follows from the results that for small SNR values the error rate is quite large and ISI is produced due the relative high power of noise. As SNR is increased the eye pattern for the received constellation gets closer to the eye pattern in an ideal channel, e.g. ISI introduced by the noise, is decreased.

Finally, the parallel binary streams are multiplexed into a serial stream, $s[n]$, and the stream of binary digits is the recovered transmitted information.

\section{Conclusions}

In this paper the basic processing involved in the receiver of the OFDM system is described and simulated. Matlab simulation results for the $2 \mathrm{k}$ mode of the DVB-T standard are obtained and presented. Simulation results correspond to the theoretical ones.

The next objective of this work is to simulate Bit Error Rate (BER) and Peak to Average Power Ratio (PAPR) for FFT based OFDM system, as well as, to study and simulate of OFDM performance over a multipath channel.

\section{References}

[1] Edfors, O., M. Sandell , J.J.Van de Beek, D. Landström, and F. Sjöberg, An Introduction to Orthogonal Frequency Division Multiplexing, Luleå, Sweden: Luleå Tekniska Universitet, 1996.

[2] Taub, H., D. L. Schilling, and G. Saha, Taub's Principles of Communication Systems, Tata McGraw Hill, 2010.

[3] Slar, B., Digital Communications: Fundamentals and Applications, Prentice-Hall, $2^{\text {nd }}$ Edition, 2001.

[4] Bahai, A.R. S., B. R. Saltzberg, and M. Ergen, Multicarrier Digital Communications: Theory and Applications of OFDM. Springer, New York, 2004.

[5] Nee, R. V., and R. Prasad, OFDM Wireless Multimedia Communications. Norwood, MA, Artech House, 2000.

[6] R. Prasad, OFDM for Wireless Communications Systems. Artech House, 2004.

[7] Bingham, J. A. C., "Multi Carrier Modulation for Data Transmission. An idea whose time has come", IEEE Communications Magazine, vol.28, no.5, pp. 5-14, May, 1990.

[8] ETS 300744 - Digital Video Broadcasting (DVB); Framing structure, channel coding and modulation for Digital Terrestrial Television, (DVB-T).

[9] онов, К., Цифрово радио и телевизионно разпръскване, Диодс, София, 2011.

[10] Sadinov, S., P. Daneva, and P. Kogias, "OFDM (Orthogonal Frequency Division Multiplexing) Transmission Simulation", International Scientific Conference Unitech 2013, Gabrovo, vol. II, pp. 121 - 126, 22 - 23 November, 2013.

[11] Pathak, N., "OFDM (Orthogonal Frequency Division Multiplexing) SIMULATION USING MATLAB", IJERT, Vol. 1, Issue 6, pp. 1-6, August - 2012.

[12] Ghorpade, S. S., and S. V. Sankpal, "Behaviour of OFDM system using MATLAB simulation", International Journal of Advanced Computer Research, vol. 3, pp. 67 - 71, June 2013. 